KARol Dziedziul (Gdańsk)

\title{
APPLICATION OF MAZUR-ORLICZ'S THEOREM IN AMISE CALCULATION
}

Abstract. An approximation error and an asymptotic formula are given for shift invariant operators of polynomial order $\varrho$. Density estimators based on shift invariant operators are introduced and AMISE is calculated.

1. Asymptotic formulas. We assume that $F, G: \mathbb{R}^{d} \rightarrow \mathbb{R}$ are functions such that there are constants $C>0$ and $0<q<1$ such that for all $x \in \mathbb{R}^{d}$,

$$
|F(x)|<C q^{|x|} \quad \& \quad|G(x)|<C q^{|x|},
$$

where $|x|^{2}=x \cdot x$ and $x \cdot x$ is the scalar product in $\mathbb{R}^{d}$. Consider the operator given by

$$
Q f(x)=\int_{\mathbb{R}^{d}} K(x, y) f(y) d y
$$

where

$$
K(x, y)=\sum_{\alpha \in \mathbb{Z}^{d}} F(y-\alpha) G(x-\alpha)
$$

For $h>0$, define

$$
Q_{h}=\sigma_{h} \circ Q \circ \sigma_{1 / h}
$$

where

$$
\sigma_{h} f(x)=f(x / h) .
$$

We call the operators with kernel of type (3) shift invariant. Examples of such operators are:

- spline operators: the Ciesielski-Durrmeyer operator (see [C]), a quasiprojection (see [Dz1]), an orthogonal projection (see [BD2], [BHR]),

2000 Mathematics Subject Classification: 41A35, 41A60, 62G07, 62G20.

Key words and phrases: shift invariant operators, asymptotic formula, density estimators, central limit, AMISE, asymptotic mean integral square error. 
- an orthogonal projection based on multiresolution approximation $[\mathrm{M}]$, operators based on shift invariant spaces (see [JZ] and [BDR]; in particular shift invariant spaces constructed by a function which satisfies the StrangFix conditions, see $[\mathrm{SF}]$ ).

Let $W_{p}^{r}$ be a Sobolev space (for details see $[\mathrm{M}]$ ). Let $C_{0}^{r}$ be the space of $r$-differentiable functions with compact support. Set

$$
\begin{gathered}
|f|_{r, p}=\sum_{|\beta|=r}\left\|D^{\beta} f\right\|_{p}, \quad\|f\|_{p}=\left(\int_{\mathbb{R}^{d}}|f|^{p}\right)^{1 / p} \\
D^{\beta} f=\frac{\partial^{|\beta|} f}{\partial x_{1}^{\beta_{1}} \ldots \partial x_{d}^{\beta_{d}}}, \quad \beta=\left(\beta_{1}, \ldots, \beta_{d}\right), \quad|\beta|=\beta_{1}+\ldots+\beta_{d} .
\end{gathered}
$$

Assume the operator $Q$ reproduces all polynomials of degree less than $r$, i.e. $Q(P)=P$ provided $\operatorname{deg} P<r$. We then say that $Q$ has polynomial order $r$. The following theorem is a generalization of [BHR, Proposition 4, p. 63].

Theorem 1.1. Let $1 \leq p<\infty$. Assume that $Q$ has polynomial order $r$. Then there is a constant $C(p)>0$ such that for all $f \in W_{p}^{r}\left(\mathbb{R}^{d}\right)$,

$$
\left\|Q_{h} f-f\right\|_{p} \leq C(p) h^{r}|f|_{r, p},
$$

Proof. Since the operators $Q_{h}$ are bounded from $L^{p}$ to $L^{p}$ it is sufficient to prove (5) for $f \in C_{0}^{r}$. Let $f \in C_{0}^{r}$. Let $P_{x}$ be the Taylor polynomial of $f$ of degree $r-1$ at $x$. Note that $f(x)=P_{x}(x)$ and $Q_{h} f(x)-f(x)=Q_{h}\left(f-P_{x}\right)(x)$. Now Lemma 1.1 below yields (5) for $1 \leq p<\infty$.

An easy computation shows the assertion for $p=\infty$ (see proof of $[\mathrm{Dz} 4$, Theorem 9.7]).

In statistics we need an asymptotic formula for the error in shift invariant operators. Such a formula was proved in [BD3], [BD4] for an interpolation operator and an orthogonal projection. Those proofs are based on a generalization of Mazur-Orlicz's theorem (see [BD3]). This theorem goes back to L. Fejér. Recall that a function $g$ defined on $\mathbb{R}^{d}$ is called $\mathbb{Z}^{d}$-periodic if for all $x \in \mathbb{R}^{d}$,

$$
g(x)=g(x+\alpha) \quad \text { for all } \alpha \in \mathbb{Z}^{d} .
$$

Theorem 1.2 (Mazur-Orlicz [MO]). If for $j=1, \ldots, m, g_{j}$ are measurable, bounded, $\mathbb{Z}^{d}$-periodic functions and $f_{j}$ are measurable functions with

$$
\int_{\mathbb{R}^{d}}\left|f_{j}(x)\right|^{p} d x<\infty
$$

for some $1 \leq p<\infty$, then

$$
\int_{\mathbb{R}^{d}}\left|\sum_{j=1}^{m} f_{j}(x) g_{j}(x / h)\right|^{p} d x \rightarrow \int_{[0,1)^{d}} \int_{\mathbb{R}^{d}}\left|\sum_{j=1}^{m} f_{j}(t) g_{j}(x)\right|^{p} d t d x \quad \text { as } h \rightarrow 0 .
$$


Earlier results concerning the asymptotic formula can be found in $[\mathrm{C}]$, [Dz1] for spline operators, and in [DU], [BD2] for an orthogonal projection in $L^{2}$. See also [DLP]. Let []$^{\beta}(x)=x^{\beta}=x_{1}^{\beta_{1}} \ldots x_{d}^{\beta_{d}}$. We present a new and simpler proof of the asymptotic formula for the error in shift invariant operators.

Theorem 1.3. Assume that $Q$ has maximal polynomial order $\varrho$. Let $1 \leq$ $p<\infty$ and $f \in W_{p}^{\varrho}\left(\mathbb{R}^{d}\right)$. Then

(8) $\lim _{h \rightarrow 0^{+}}\left\|\frac{Q_{h} f-f}{h^{\varrho}}\right\|_{p}^{p}=\int_{\mathbb{R}^{d}}\left(\int_{[0,1]^{d}}\left|\sum_{|\beta|=\varrho} \frac{1}{\beta !} D^{\beta} f(t)\left(Q\left([]^{\beta}\right)(x)-x^{\beta}\right)\right|^{p} d x\right) d t$.

Proof. It is sufficient to prove (8) for the dense subset $C_{0}^{\varrho+1}$ of $W_{p}^{\varrho}\left(\mathbb{R}^{d}\right)$ since

$$
\left\|\frac{Q_{h} f-f}{h^{\varrho}}\right\|_{p} \leq C|f|_{\varrho, p} .
$$

Fix $f \in C_{0}^{\varrho+1}$. Let $P_{x}$ be the Taylor polynomial of degree $\varrho$ of $f$ at $x$. By the triangle inequality (we take $F(x)=Q_{h}\left(P_{x}\right)(x) \neq P_{x}(x)$ )

$$
\left\|\frac{Q_{h} f-f}{h^{\varrho}}\right\|_{p} \leq\left\|\frac{Q_{h}(f-P .)}{h^{\varrho}}\right\|_{p}+\left\|\frac{Q_{h} P .-P .}{h^{\varrho}}\right\|_{p}
$$

and

$$
\left\|\frac{Q_{h} P .-P .}{h^{\varrho}}\right\|_{p} \leq\left\|\frac{Q_{h} f-f}{h^{\varrho}}\right\|_{p}+\left\|\frac{Q_{h}(f-P .)}{h^{\varrho}}\right\|_{p} .
$$

If we prove that there is $C$ such that for all $f \in C_{0}^{\varrho+1}$,

$$
\left\|Q_{h}(f-P .)\right\|_{p} \leq C h^{\varrho+1}|f|_{\varrho+1, p},
$$

then the proof of (8) is completed by showing that

$$
\begin{aligned}
& \lim _{h \rightarrow 0^{+}}\left\|\frac{Q_{h} P .-P .}{h^{\varrho}}\right\|_{p}^{p} \\
& \quad=\int_{\mathbb{R}^{d}}\left(\int_{[0,1]^{d}}\left|\sum_{|\beta|=\varrho} \frac{1}{\beta !} D^{\beta} f(t)\left(Q\left([]^{\beta}\right)(x)-x^{\beta}\right)\right|^{p} d x\right) d t .
\end{aligned}
$$

The technical proof of (9) is postponed to Lemma 1.1. Let

$$
P_{x}=T_{x}+R_{x},
$$

where $T_{x}$ is homogeneous of degree $\varrho$ and $\operatorname{deg} R_{x}<\varrho$. Since $Q\left(R_{x}\right)=R_{x}$ we have

$$
\begin{aligned}
\frac{Q_{h}\left(P_{x}\right)(t)-P_{x}(t)}{h^{\varrho}} & =\frac{Q_{h}\left(T_{x}\right)(t)-T_{x}(t)}{h^{\varrho}}=Q\left(T_{x}\right)(t / h)-T_{x}(t / h) \\
& =\sum_{|\beta|=\varrho} \frac{1}{\beta !} D^{\beta} f(x)\left(Q\left([]^{\beta}\right)(t / h)-(t / h)^{\beta}\right) .
\end{aligned}
$$


Consequently, from (11) we get

$$
\left\|\frac{Q_{h} P .-P .}{h \varrho}\right\|_{p}^{p}=\int_{\mathbb{R}^{d}}\left|\sum_{|\beta|=\varrho} \frac{1}{\beta !} D^{\beta} f(x)\left(Q\left([]^{\beta}\right)(x / h)-(x / h)^{\beta}\right)\right|^{p} d x .
$$

An easy calculation shows (cf. [Dz3, Lemma 3.3]) that the functions

$$
Q\left([]^{\beta}\right)(x)-x^{\beta}=(-1)^{|\beta|} \sum_{\alpha \in \mathbb{Z}^{d}} \int_{\mathbb{R}^{d}}(x-y)^{\beta} F(y-\alpha) d y G(x-\alpha)
$$

are $\mathbb{Z}^{d}$-periodic. Now the Mazur-Orlicz Theorem (7) implies (10).

Lemma 1.1. Let $1 \leq p<\infty$. Let $P_{x}$ be the Taylor polynomial of degree $k-1$ of a function $f$. There is $C$ such that for all $f \in C_{0}^{k}$,

$$
\left\|Q_{h}(f-P .)\right\|_{p} \leq C h^{k}|f|_{k, p} \text {. }
$$

Proof. By Taylor's formula,

$$
\begin{aligned}
\left\|Q_{h}(f-P .)\right\|_{p}^{p}= & \int_{\mathbb{R}^{d}} \mid \sum_{\alpha \in \mathbb{Z}^{d}} \int_{\mathbb{R}^{d}} \int_{0}^{1} \sum_{|\beta|=k} \frac{1}{\beta !} D^{\beta} f(x+s(h y-x))(1-s)^{k-1} d s \\
& \times\left.(h y-x)^{\beta} F(y-\alpha) d y G(x / h-\alpha)\right|^{p} d x .
\end{aligned}
$$

To prove (12), using assumption (1), it is sufficient to estimate

$$
\begin{aligned}
J_{\beta}= & \int_{\mathbb{R}^{d}}\left|\sum_{\alpha \in \mathbb{Z}^{d}} \int_{\mathbb{R}^{d}} \int_{0}^{1}\right| D^{\beta} f(x+s(h y-x)) \mid d s \\
& \times\left.|h y-x|^{k} q^{|y-\alpha|} d y q^{|x / h-\alpha|}\right|^{p} d x .
\end{aligned}
$$

We apply Jensen's inequality three times:

$$
\begin{aligned}
\left(\int_{0}^{1} g(s) d s\right)^{p} & \leq \int_{0}^{1}|g(x)|^{p} d x \\
\left(\sum_{\alpha \in \mathbb{Z}^{d}}\left|a_{\alpha}\right| q^{|x-\alpha|}\right)^{p} & \leq C_{1} \sum_{\alpha \in \mathbb{Z}^{d}}\left|a_{\alpha}\right|^{p} q^{|x-\alpha|},
\end{aligned}
$$

where $C_{1}$ is independent of $x$, i.e. $C_{1}=\max _{x}\left(\sum_{\alpha \in \mathbb{Z}^{d}} q^{|x-\alpha|}\right)^{p-1}$,

$$
\left(\int_{\mathbb{R}^{d}}|g(y)| q^{|y-\alpha|} d y\right)^{p} \leq C_{2} \int_{\mathbb{R}^{d}}|g(y)|^{p} q^{|y-\alpha|} d y,
$$

where $C_{2}$ is independent of $\alpha$. Consequently,

$$
\begin{aligned}
J_{\beta} \leq & C \sum_{\alpha \in \mathbb{Z}^{d}} \int_{\mathbb{R}^{d}} \int_{\mathbb{R}^{d}} \int_{0}^{1}\left|D^{\beta} f(x+s(h y-x))\right|^{p} d s \\
& \times|h y-x|^{p k} q^{|y-\alpha|} d y q^{|x / h-\alpha|} d x .
\end{aligned}
$$


Letting $x / h-\alpha=u$ yields

$$
\begin{aligned}
J_{\beta} \leq & C h^{d} \sum_{\alpha \in \mathbb{Z}^{d}} \int_{\mathbb{R}^{d}} \int_{\mathbb{R}^{d}} \int_{0}^{1}\left|D^{\beta} f(h u+h \alpha+s(h y-h u-h \alpha))\right|^{p} d s \\
& \times|h y-h u-h \alpha|^{p k} q^{|y-\alpha|} d y q^{|u|} d u
\end{aligned}
$$

and by obvious changes of variables

$$
\begin{aligned}
J_{\beta} \leq & C h^{d} \sum_{\alpha \in \mathbb{Z}^{d}} \int_{\mathbb{R}^{d}} \int_{\mathbb{R}^{d}} \int_{0}^{1}\left|D^{\beta} f(h u+h \alpha+\operatorname{sh}(z-u))\right|^{p} d s \\
& \times h^{p k}|z-u|^{p k} q^{|z|} d z q^{|u|} d u \\
= & C h^{d+p k} \sum_{\alpha \in \mathbb{Z}^{d}} \int_{\mathbb{R}^{d}} \int_{\mathbb{R}^{d}}^{1}\left|D_{0}^{\beta} f(h u+h \alpha+s h v)\right|^{p} d s|v|^{p k} q^{|u+v|} d v q^{|u|} d u .
\end{aligned}
$$

Let us split the integrals:

$$
\begin{aligned}
J_{\beta} \leq & C h^{d+p k} \sum_{n=1}^{\infty} \sum_{\alpha \in \mathbb{Z}^{d}} \int_{n-1<|u|<n} \int_{\mathbb{R}^{d}} \int_{0}^{1}\left|D^{\beta} f(h u+h \alpha+s h v)\right|^{p} d s \\
& \times|v|^{p k} q^{|u+v|} d v q^{n-1} d u \\
\leq & C h^{d+p k} \sum_{n=1}^{\infty} q^{n-1} \sum_{\alpha \in \mathbb{Z}^{d}} \int_{n-1<|u|<n} \sum_{j=1}^{\infty} q^{j-1} \\
& \times \int_{j-1<|u+v|<j} \int_{0}^{1}\left|D^{\beta} f(h u+h \alpha+s h v)\right|^{p} d s|v|^{p k} d v d u .
\end{aligned}
$$

Note that if $|v+u|<j$ and $|u|<n$ then

$$
|v|<|v+u|+|u|<j+n \text {. }
$$

Thus

$$
\begin{aligned}
J_{\beta} \leq & C h^{d+p k} \sum_{n=1}^{\infty} q^{n-1} \sum_{\alpha \in \mathbb{Z}^{d}} \int_{|u|<n} \sum_{j=1}^{\infty} q^{j-1} \\
& \times \int_{|v|<j+n} \int_{0}^{1}\left|D^{\beta} f(h u+h \alpha+s h v)\right|^{p} d s|v|^{p k} d v d u .
\end{aligned}
$$

Changing the order of the integrations we get

$$
\begin{aligned}
J_{\beta} \leq & C h^{d+p k} \sum_{n=1}^{\infty} q^{n-1} \sum_{j=1}^{\infty} q^{j-1} \\
& \times \int_{|v|<j+n} \int_{0}^{1} \sum_{\alpha \in \mathbb{Z}^{d}} \int_{|u|<n}\left|D^{\beta} f(h u+h \alpha+s h v)\right|^{p} d u d s|v|^{p k} d v .
\end{aligned}
$$


Note that if $|v|<j+n$ then

$$
\begin{aligned}
& h^{d} \sum_{\alpha \in \mathbb{Z}^{d}} \int_{|u|<n}\left|D^{\beta} f(h u+h \alpha+s h v)\right|^{p} d u \\
& \quad \leq \sum_{\alpha \in \mathbb{Z}^{d}} \int_{|x-h \alpha|<h(2 n+j)}\left|D^{\beta} f(x)\right|^{p} d x \leq(4 n+2 j)^{d} \int_{\mathbb{R}^{d}}\left|D^{\beta} f(x)\right|^{p} d x
\end{aligned}
$$

and moreover

$$
\int_{|v|<j+n}|v|^{p k} d v=C(j+n)^{p k+d}
$$

Consequently,

$$
\begin{aligned}
J_{\beta} & \leq C \sum_{n=1}^{\infty} q^{n-1} h^{p k} \sum_{j=1}^{\infty} q^{j-1}(4 n+2 j)^{d}(j+n)^{p k+d} \int_{\mathbb{R}^{d}}\left|D^{\beta} f(x)\right|^{p} d x \\
& \leq C h^{p k} \int_{\mathbb{R}^{d}}\left|D^{\beta} f\right|^{p} .
\end{aligned}
$$

This finishes the proof of the lemma.

Let $X_{1}, \ldots, X_{n}$ be a random sample from a distribution with density $f \in W_{2}^{\varrho}$. We define a density estimator based on the kernel $K$ by

$$
f_{h, n}(x)=\frac{1}{n} \sum_{j=1}^{n} K_{h}\left(x, X_{j}\right)
$$

where

$$
K_{h}(x, y)=(1 / h)^{d} K(x / h, y / h)
$$

Note that

$$
E f_{h, n}=Q_{h} f
$$

As usual we consider the estimation error given by

$$
\operatorname{MISE}(f, h)=E\left[\int_{\mathbb{R}^{d}}\left[f_{h, n}-f\right]^{2}\right] \text {. }
$$

It is known that

$$
\operatorname{MISE}(f, h)=E\left[\int_{\mathbb{R}^{d}}\left[f_{h, n}-Q_{h} f\right]^{2}\right]+\int_{\mathbb{R}^{d}}\left[Q_{h} f-f\right]^{2} .
$$

The asymptotic formula for the second factor in (16) is given in (8). We prove that

THEOREM 1.4. Assume that $Q$ has maximal polynomial order $\varrho>0$. If $n h^{d} \rightarrow \infty, h \rightarrow 0$ then

$$
\lim _{n h^{d} \rightarrow \infty} n h^{d} E\left[\int_{\mathbb{R}^{d}}\left[f_{h, n}-Q_{h} f\right]^{2}\right]=\int_{\mathbb{R}^{d}}\left[\int_{[0,1]^{d}} K^{2}(x, y) d y\right] d x,
$$


where

$$
\int_{\mathbb{R}^{d}}\left[\int_{[0,1]^{d}} K^{2}(x, y) d y\right] d x=\sum_{\alpha \in \mathbb{Z}^{d}} \eta(\alpha) \xi(\alpha)
$$

and

$$
\eta=G * \breve{G}, \quad \xi=F * \breve{F}, \quad \breve{G}(x)=G(-x), \quad \breve{F}(x)=F(-x) .
$$

Proof. Note that

$$
\begin{aligned}
E\left[\int_{\mathbb{R}^{d}}\left[f_{h, n}-E f_{h, n}\right]^{2}\right] & =\frac{1}{n^{2}} \sum_{j=1}^{n} \int_{\mathbb{R}^{d}} E\left[K_{h}\left(x, X_{j}\right)-E K_{h}\left(x, X_{j}\right)\right]^{2} d x \\
& =\frac{1}{n^{2}} \sum_{j=1}^{n} \int_{\mathbb{R}^{d}}\left(E\left[K_{h}^{2}\left(x, X_{j}\right)\right]-\left[E K_{h}\left(x, X_{j}\right)\right]^{2}\right) d x .
\end{aligned}
$$

If $h \rightarrow 0$ then by (5),

$$
\int_{\mathbb{R}^{d}}\left[E K_{h}\left(x, X_{j}\right)\right]^{2} d x=\int_{\mathbb{R}^{d}}\left(Q_{h} f\right)^{2} \rightarrow \int_{\mathbb{R}^{d}} f^{2} .
$$

On the other hand

$$
\int_{\mathbb{R}^{d}} E K_{h}^{2}\left(x, X_{j}\right) d x=\iint_{\mathbb{R}^{d}} K_{\mathbb{R}^{d}}^{2}(x, y) f(y) d y d x .
$$

From Fubini's theorem

$$
\int_{\mathbb{R}^{d}} E K_{h}^{2}\left(x, X_{j}\right) d x=\frac{1}{h^{d}} \int_{\mathbb{R}^{d}}\left[\int_{\mathbb{R}^{d}} K^{2}(u, y / h) d u\right] f(y) d y .
$$

Note that for all $\alpha \in \mathbb{Z}^{d}$,

$$
\int_{\mathbb{R}^{d}} K^{2}(x, y+\alpha) d x=\int_{\mathbb{R}^{d}} K^{2}(x, y) d x .
$$

From Mazur-Orlicz's theorem we get

$$
\lim _{h \rightarrow 0} \int_{\mathbb{R}^{d}}\left[\int_{\mathbb{R}^{d}} K^{2}(u, y / h) d u\right] f(y) d y=\int_{[0,1]^{d}}\left[\int_{\mathbb{R}^{d}} K^{2}(u, y) d u\right] d y \int_{\mathbb{R}^{d}} f(y) d y .
$$

We thus get (17). A simple calculation leads to (18).

Remarks. 1. From (16)-(8) we get

$$
\begin{aligned}
\operatorname{MISE}(f, h) \sim \mathrm{AMISE} & :=\frac{1}{n h^{d}} \int_{\mathbb{R}^{d}}\left[\int_{[0,1]^{d}} K^{2}(x, y) d y\right] d x \\
& +h^{2 \varrho} \int_{\mathbb{R}^{d}}\left(\int_{[0,1]^{d}}\left|\sum_{|\beta|=\varrho} \frac{1}{\beta !} D^{\beta} f(t) Q\left([]^{\beta}\right)(x)-x^{\beta}\right|^{2} d x\right) d t .
\end{aligned}
$$


So the best choice of $h>0$ which minimizes (16) is

$$
h \sim n^{-1 /(2 \varrho+d)} .
$$

2. Using the methods of [Dz2] one can prove the central limit theorem. This theorem generalizes the results for wavelet estimators [DL1]-[DL2] in $\mathbb{R}^{d}$ and box spline estimators [Dz2]. These results are motivated by the result for the Rosenblatt-Parzen estimator $[\mathrm{H}]$.

\section{References}

[BD1] M. Beśka and K. Dziedziul, The saturation theorem for interpolation and the Bernstein-Schnabl operator, Math. Comp. 70 (2001), 705-717.

[BD2] - - - The saturation theorem for orthogonal projection, in: Advances in Multivariate Approximation, W. Haussmann et al. (eds.), Wiley, 1999, 73-83.

[BD3] -, -, Asymptotic formula for the error in cardinal interpolation, Numer. Math. 89 (2001), 445-456.

[BD4] - - - Asymptotic formula for the error in orthogonal projection, Math. Nachr. 233 (2002), 47-53.

[BDR] C. de Boor, R. A. DeVore and A. Ron, Approximation from shift-invariant subspaces of $L_{2}\left(\mathbb{R}^{d}\right)$, Trans. Amer. Math. Soc. 341 (1994), 787-806.

[BHR] C. de Boor, K. Höllig and S. Riemenschneider, Box Splines, Springer, 1993.

[C] Z. Ciesielski, Asymptotic nonparametric spline density estimation in several variables, in: Internat. Ser. Numer. Math. 94, Birkhäuser, Basel, 1990, 25-53.

[DU] I. Daubechies and M. Unser, On the approximation power of convolution-based least squares versus interpolation, IEEE Trans. Signal Process. 45 (1997), 16971711.

[DL1] P. Doukhan and J. R. León, Quadratic deviation of projection density estimates, Rebrape 7 (1993), 37-63.

[DL2] -, 一, Déviation quadratique d'estiimateurs de densité par projections orthogonales, C. R. Acad. Sci. Paris Sér. I Math. 310 (1990), 425-430.

[DLP] P. Doukhan, J. Léon et F. Portal, Une mesure de la déviation quadratique d'estimateurs non paramétriques, Ann. Inst. H. Poincaré Probab. Statist. 22 (1986), $37-66$.

[Dz1] K. Dziedziul, The saturation theorem for quasi-projections, Studia Sci. Math. Hungar. 35 (1999), 99-111.

[Dz2] -, Central limit theorem for square error of multivariate nonparametric box spline density estimators, Appl. Math. (Warsaw) 28 (2001), 437-456.

[Dz3] -, Asymptotic formulas in cardinal interpolation and orthogonal projection, in: Recent Progress in Multivariate Approximation, W. Haussmann et al. (eds.), Internat. Ser. Numer. Math. 137, Birkhäuser, Basel, 2001, 139-157.

[Dz4] —, Box Splines, Wyd. Politechniki Gdańskiej, 1997 (in Polish).

$[\mathrm{H}] \quad$ P. Hall, Central limit theorem for integrated square error of multivariate nonparametric density estimators, J. Multivariate Anal. 14 (1984), 1-16.

[JZ] K. Jetter and D.-X. Zhou, Order of linear approximation from shift-invariant spaces, Constr. Approx. 11 (1995), 423-438.

[MO] S. Mazur et W. Orlicz, Sur quelques propriétés de fonctions périodiques, Studia Math. 9 (1940), 1-16.

[M] Y. Meyer, Wavelets and Operators, Cambridge Univ. Press, Cambridge, 1992. 
[SF] G. Strang and G. Fix, A Fourier analysis of the finite element variational method, in: Constructive Aspects of Functional Analysis, G. Geymonat (ed.), Ed. Cremonese, Roma, 1973, 793-840.

Faculty of Applied Mathematics

Technical University of Gdańsk

G. Narutowicza 11/2

80-952 Gdańsk, Poland

E-mail: kdz@mifgate.pg.gda.pl

Received on 5.11.2001;

revised version on 22.3.2002 